Supporting Information

\title{
Long-Term Lysosomes Tracking with A Water-Soluble Two-Photon Phosphorescent Iridium(III) Complex
}

\author{
Kangqiang Qiu, Huaiyi Huang, Bingyang Liu, Yukang Liu, Ziyi Huang, \\ Yu Chen, Liangnian Ji, and Hui Chao* \\ MOE Key Laboratory of Bioinorganic and Synthetic Chemistry, School of Chemistry and \\ Chemical Engineering, Sun Yat-Sen University, Guangzhou 510275, P. R. China \\ Tel: +86-20-84110613; Fax: +86-20-84112245; Email: ceschh@mail.sysu.edu.cn
}




\section{Table of Contents}

Figure S1 ESI-MS spectrum of Ir-lyso.............................................................

Figure S2 ${ }^{1}$ H NMR spectrum of Ir-lyso...............................................................

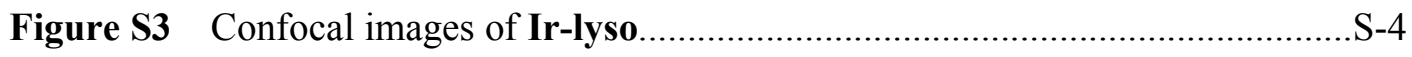

Figure S4 One-photon imaging of Ir-lyso in 3D multicellular spheroids..................S-5

Table S1 Crystal data and structural refinements for Ir-lyso....................................

Table S2 Selected bond lengths and angles for Ir-lyso.........................................

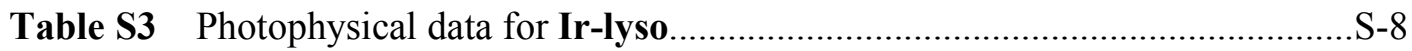




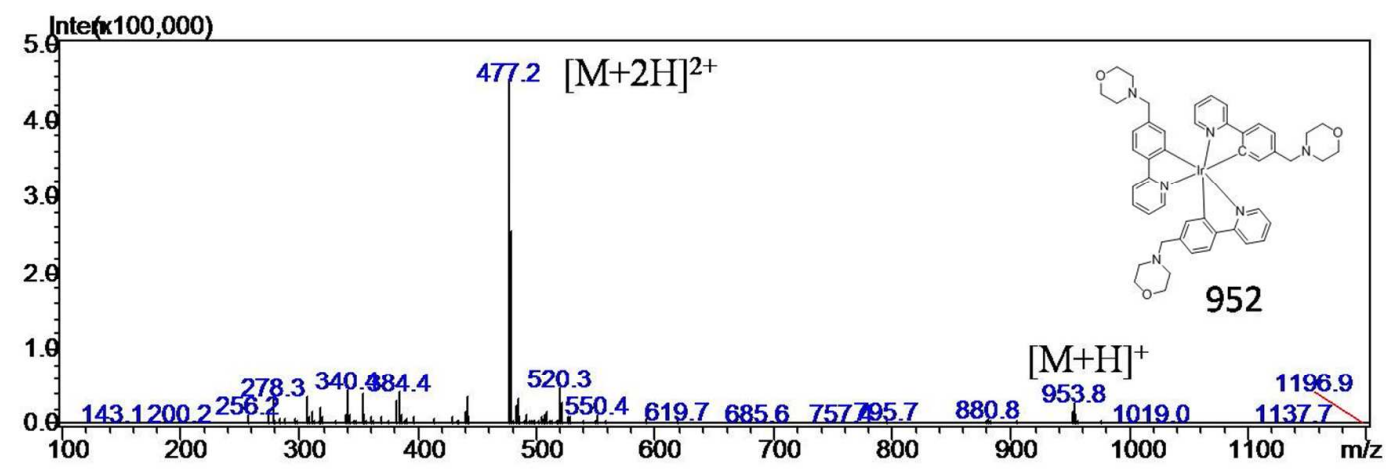

Figure S1. ESI-MS spectrum of Ir-lyso. 


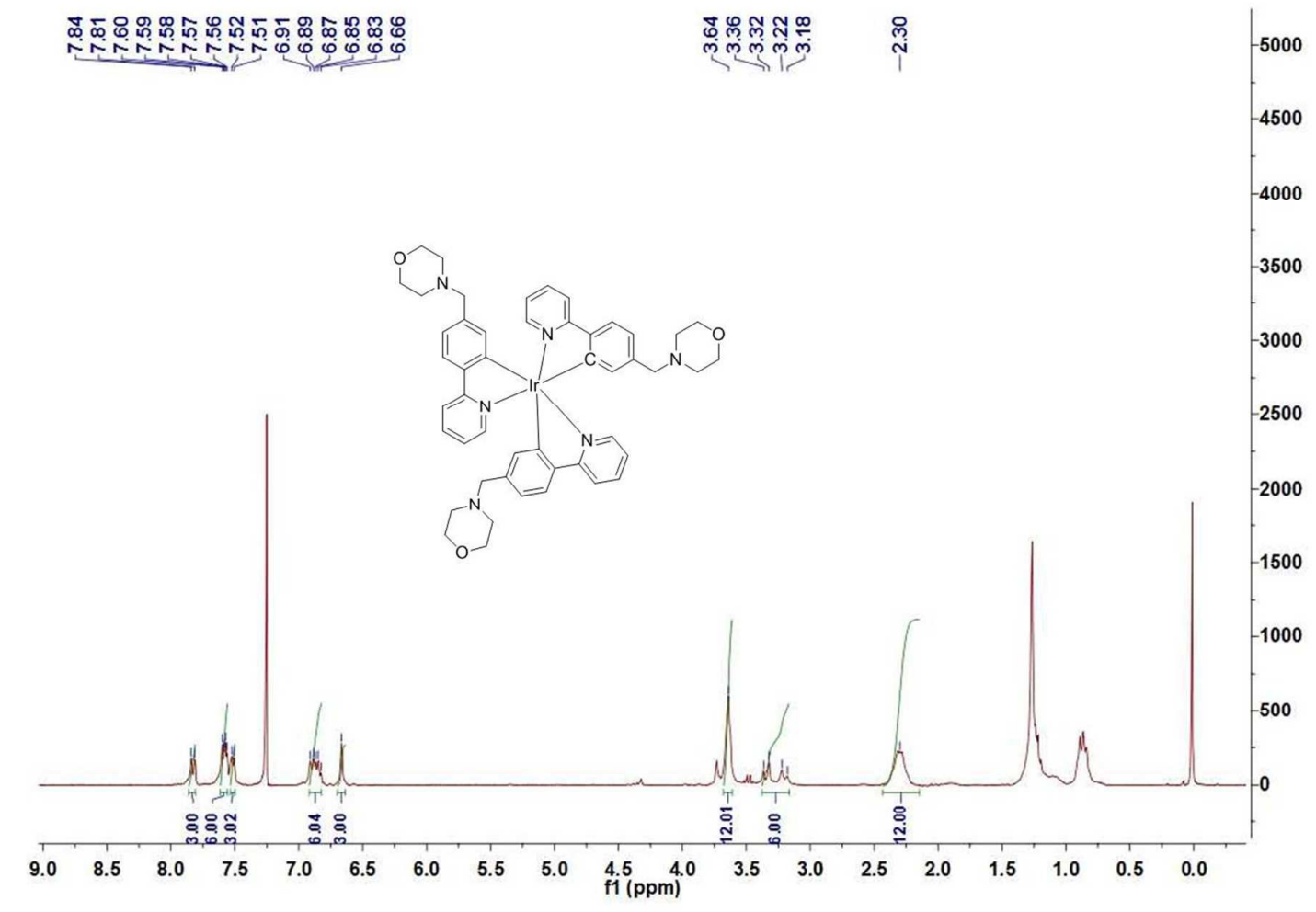

Figure S2. $\quad{ }^{1}$ H NMR spectrum of Ir-lyso. 

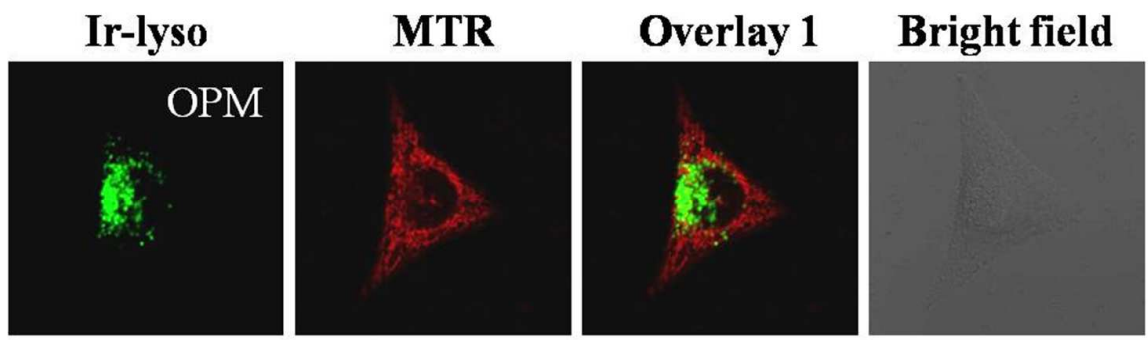

\section{Overlay 2}
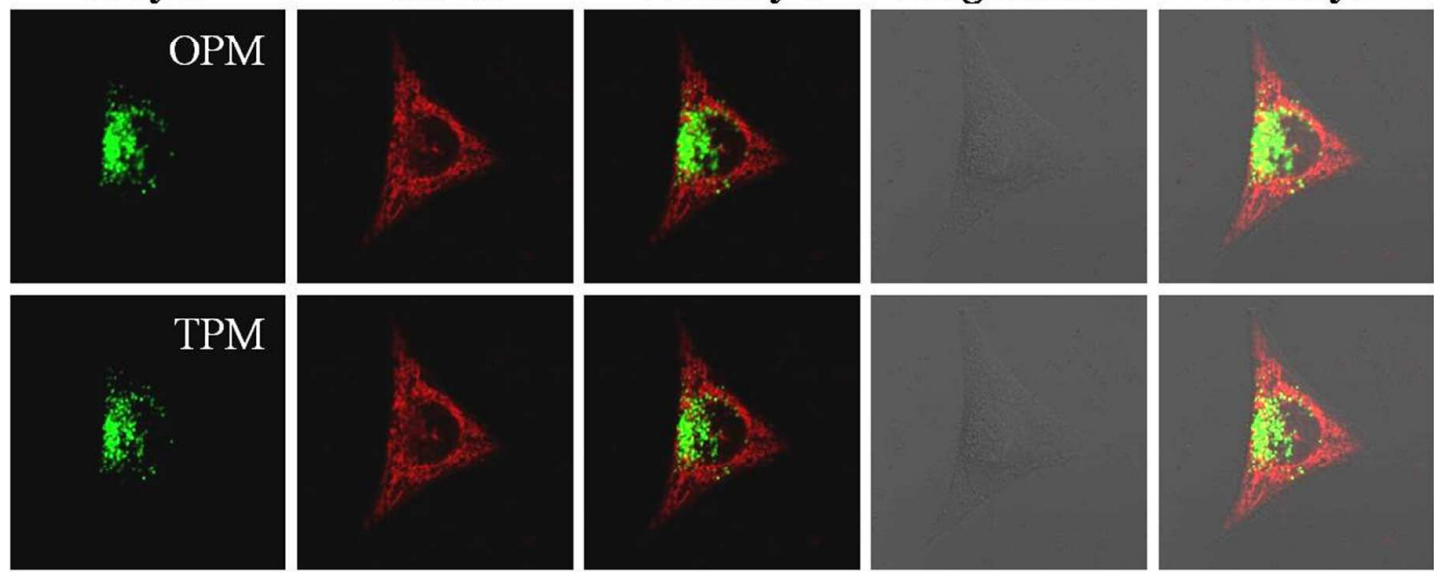

Figure S3. One-photon microscopy (OPM) and two-photon microscopy (TPM) images of HeLa cells co-labeled with Ir-lyso ( $2 \mu \mathrm{M}, 0.5$ h) and MTR ( $50 \mathrm{nM}, 0.5$ h). Ir-lyso was excited at $405 \mathrm{~nm}(\mathrm{OPM})$ or $750 \mathrm{~nm}(\mathrm{TPM})$. MTR (OPM) was excited at $543 \mathrm{~nm}$. The phosphorescence/fluorescence was collected at $520 \pm 20 \mathrm{~nm}$ and $620 \pm 20 \mathrm{~nm}$ for Ir-lyso and MTR, respectively. Overlay 1: Overlay of the $1^{\text {st }}$ and $2^{\text {nd }}$ columns. Overlay 2 : Overlay of the $3^{\text {rd }}$ and $4^{\text {th }}$ columns. 


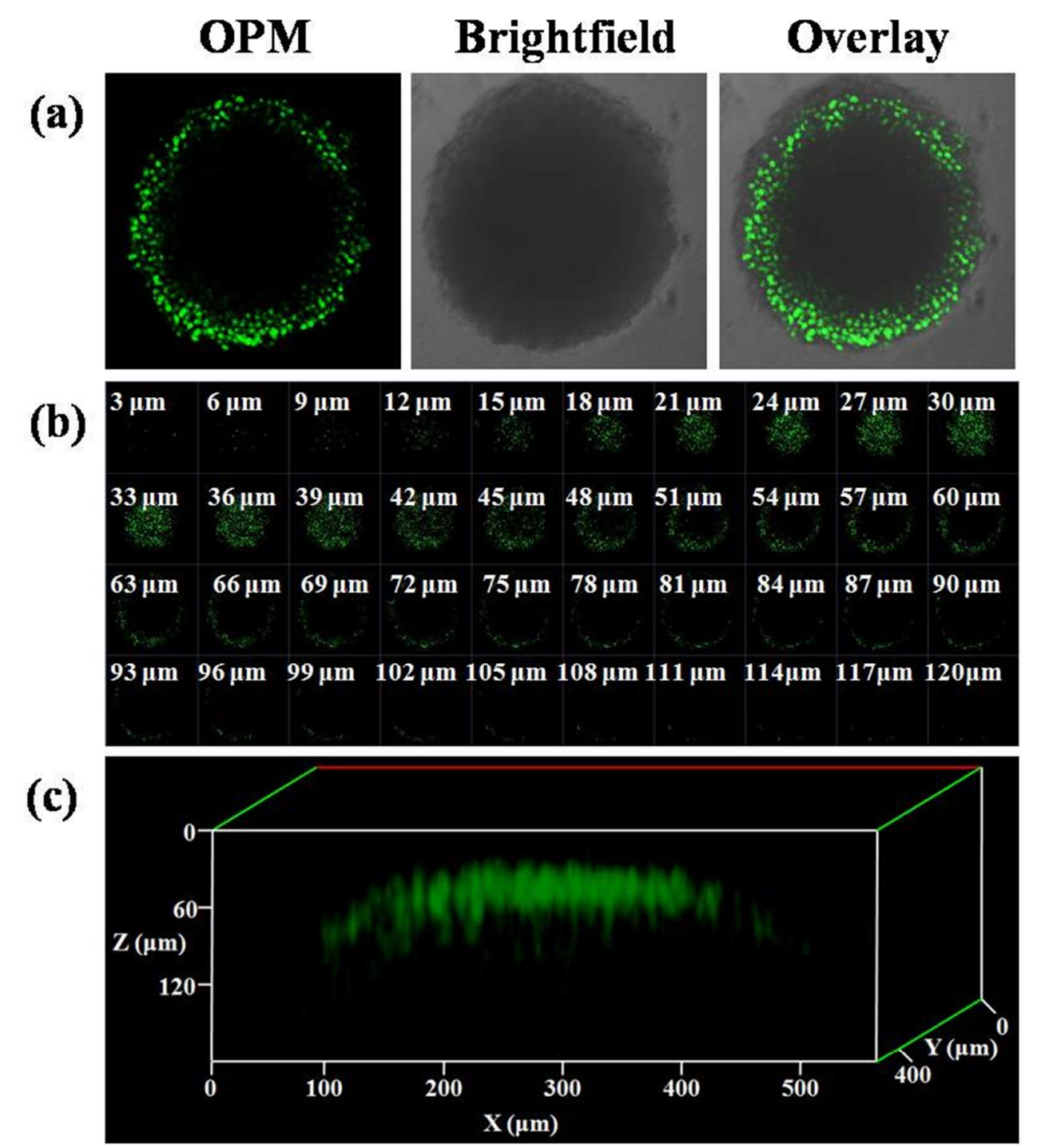

Figure S4. (a) One-photon phosphorescent images of 3D tumor spheroids after incubation with Ir-lyso $(2 \mu \mathrm{M})$ for $6 \mathrm{~h}$. (b) The one-photon Z-stack images were taken of every $3 \mu \mathrm{m}$ section from the top to bottom. (c) The one-photon 3D Z-stack images of an intact spheroid. The images were taken under a $10 \times$ objective. $\lambda_{\mathrm{ex}}=405 \mathrm{~nm} ; \lambda_{\mathrm{em}}=520 \pm$ $20 \mathrm{~nm}$. 
Table S1 Crystal data and structural refinements for Ir-lyso

\begin{tabular}{|c|c|}
\hline Crystal data & Ir-lyso \\
\hline Empirical formula & $\mathrm{C}_{48} \mathrm{H}_{51} \mathrm{IrN}_{6} \mathrm{O}_{3}$ \\
\hline Formula weight & 952.15 \\
\hline Crystal system & Triclinic \\
\hline Temperature & $150(2) \mathrm{K}$ \\
\hline Space group & $P \overline{1}$ \\
\hline \multirow[t]{6}{*}{ Unit cell dimensions } & $\mathrm{a}=15.0306(6) \AA$ \\
\hline & $\mathrm{b}=15.0306(6) \AA$ \\
\hline & $\mathrm{c}=12.6125(10) \AA$ \\
\hline & $\alpha=90.00^{\circ}$ \\
\hline & $\beta=90.00^{\circ}$ \\
\hline & $\gamma=120.00^{\circ}$ \\
\hline Volume & $2467.7(2) \AA^{3}$ \\
\hline $\mathrm{Z}$ & 2 \\
\hline Calculated density & $1.281 \mathrm{~g} / \mathrm{cm}^{3}$ \\
\hline Absorption coefficient & $2.747 \mathrm{~mm}^{-1}$ \\
\hline$F(000)$ & 964 \\
\hline$\theta$ range for data collection $\left({ }^{\circ}\right)$ & $3.13-27.48$ \\
\hline \multirow[t]{3}{*}{ Index ranges } & $-19 \leq \mathrm{h} \leq 16$ \\
\hline & $-18 \leq \mathrm{k} \leq 19$ \\
\hline & $-12 \leq 1 \leq 16$ \\
\hline Reflections collected & 3782 \\
\hline \multirow{2}{*}{ Independent reflections } & 3446 \\
\hline & {$[\mathrm{R}($ int $)=0.0555]$} \\
\hline Data / restraints / parameters & 3782 / 6 / 175 \\
\hline \multirow{2}{*}{ Final $R$ indices $[I>2 \operatorname{sigma}(I)]$} & $\mathrm{R} 1=0.0785$ \\
\hline & $\mathrm{wR} 2=0.1935$ \\
\hline \multirow{2}{*}{$R$ indices (all data) } & $\mathrm{R} 1=0.0728$ \\
\hline & $\mathrm{wR} 2=0.1884$ \\
\hline
\end{tabular}


Table S2 Selected bond lengths $(\AA)$ and angles $\left({ }^{\circ}\right)$ for Ir-lyso

\begin{tabular}{cccccccc}
\hline Ir1-C1 & $2.024(8)$ & Ir1-N1 & $2.162(6)$ & Ir1-C1A & $2.024(8)$ & Ir1-N1A & $2.162(6)$ \\
Ir1-C1B & $2.024(8)$ & Ir1-N1B & $2.162(6)$ & C1-Ir1-C1A & $95.3(3)$ & C1-Ir1-C1B & $95.3(3)$ \\
C1A-Ir1-C1B & $95.3(3)$ & C1-Ir1-N1 & $79.4(3)$ & C1-Ir1-N1A & $90.4(3)$ & C1-Ir1-N1B & $172.6(3)$ \\
C1A-Ir1-N1 & $90.4(3)$ & C1A-Ir1-N1A & $172.6(3)$ & C1A-Ir1-N1B & $79.4(3)$ & N1-Ir1-N1A & $95.2(2)$ \\
C1B-Ir1-N1 & $172.6(3)$ & C1B-Ir1-N1A & $79.4(3)$ & C1B-Ir1-N1B & $90.4(3)$ & N1-Ir1-N1B & $95.4(2)$ \\
N1A-Ir1-N1B & $95.4(2)$ & & & & & & \\
\hline
\end{tabular}


Table S3 Photophysical data for Ir-lyso in disodium hydrogen phosphate/citric acid buffer solution $(\mathrm{pH}=5.5)$

\begin{tabular}{|c|c|c|c|c|c|c|}
\hline Complex & $\lambda_{\mathrm{ab}}{ }^{a}$ & $\varepsilon^{b}$ & $\lambda_{\mathrm{em}}{ }^{c}$ & $\varphi^{d}$ & $\tau / \mathrm{nS}^{e}$ & $\delta / \mathrm{GM}^{f}$ \\
\hline \multicolumn{7}{|c|}{$\begin{array}{l}{ }^{a} \lambda_{\mathrm{ab}} \text { maximum values of the one-photon absorption in } \mathrm{nm} .{ }^{b} \text { Extinction coefficient in } \\
1 \times 10^{4} \mathrm{M}^{-1} \cdot \mathrm{cm}^{-1} \cdot{ }^{c} \lambda_{\text {em }} \text { maximum values of the one-photon emission spectra in } \mathrm{nm} . \\
\text { Phosphorescent quantum yield. }{ }^{e} \text { Life time. }{ }^{f} \text { Two-photon absorption cross section at } 750 \\
\text { nm, measured in methanol. }\end{array}$} \\
\hline
\end{tabular}

